\title{
Properties of linear integral equations related to the six-vertex model with disorder parameter
}

\author{
Hermann Boos and Frank Göhmann \\ Fachbereich C - Physik, Bergische Universität Wuppertal, \\ 42097 Wuppertal, Germany
}

\begin{abstract}
One of the key steps in recent work on the correlation functions of the XXZ chain was to regularize the underlying six-vertex model by a disorder parameter $\alpha$. For the regularized model it was shown that all static correlation functions are polynomials in only two functions. It was further shown that these two functions can be written as contour integrals involving the solutions of a certain type of linear and non-linear integral equations. The linear integral equations depend parametrically on $\alpha$ and generalize linear integral equations known from the study of the bulk thermodynamic properties of the model. In this note we consider the generalized dressed charge and a generalized magnetization density. We express the generalized dressed charge as a linear combination of two quotients of $Q$ functions, the solutions of Baxter's $t$ - $Q$-equation. With this result we give a new proof of a lemma on the asymptotics of the generalized magnetization density as a function of the spectral parameter.
\end{abstract}

Keywords: quantum spin chains, correlation functions

\section{Introduction}

In our present understanding of the thermodynamics ${ }^{9}$ and the finite temperature correlation functions $s^{2,4,6,8}$ of the XXZ quantum spin chain certain complex valued functions defined as solutions of linear or non-linear integral equations play an important role. In first place we have to mention the so-called auxiliary function $\mathfrak{a}$, satisfying the non-linear integral equation

$$
\ln (\mathfrak{a}(\lambda \mid \kappa))=-2 \kappa \eta-\frac{2 J \operatorname{sh}(\eta) \mathrm{e}(\lambda)}{T}-\int_{C} \frac{\mathrm{d} \mu}{2 \pi \mathrm{i}} K(\lambda-\mu) \ln (1+\mathfrak{a}(\mu \mid \kappa)) .
$$

Here $J$ sets the energy scale of the spin chain, $T$ is the temperature, and $\eta$ controls the anisotropy ${ }^{\mathrm{a}}$. The bare energy $\mathrm{e}(\lambda)$ and the kernel $K(\lambda)$ are defined as

$$
\mathrm{e}(\lambda)=\operatorname{cth}(\lambda)-\operatorname{cth}(\lambda+\eta), \quad K(\lambda)=\operatorname{cth}(\lambda-\eta)-\operatorname{cth}(\lambda+\eta) .
$$

a The anisotropy parameter of the XXZ Hamiltonian is $\Delta=\operatorname{ch}(\eta)$ and the quantum group parameter $q=\mathrm{e}^{\eta}$. For simplicity we shall assume throughout that $\operatorname{Re} \eta=0$ and $0<\operatorname{Im} \eta<\pi / 2$. This means to consider the $\mathrm{XXZ}$ chain in the critical regime. 
The integration contour $C$ encircles the real axis at a distance slightly smaller than $\gamma / 2=\operatorname{Im} \eta / 2$. The twist parameter $\kappa$ is proportional to the magnetic field $h$, $\kappa=h / 2 T \eta$.

The auxiliary function $\mathfrak{a}$ determines the free energy per lattice site,

$$
f(h, T)=-\frac{h}{2}-T \int_{C} \frac{\mathrm{d} \lambda}{2 \pi \mathrm{i}} \mathrm{e}(\lambda) \ln (1+\mathfrak{a}(\lambda \mid \kappa)),
$$

of the spin chain and, hence, all its thermodynamic properties. This explains the importance of $\mathfrak{a}$.

The magnetization, for instance, is defined as

$$
m(h, T)=-\frac{\partial f(h, T)}{\partial h} .
$$

It has a simple expression in terms of the logarithmic derivative of the auxiliary function,

$$
\sigma(\lambda)=-T \partial_{h} \ln (\mathfrak{a}(\lambda \mid \kappa))
$$

namely,

$$
m(h, T)=-\frac{1}{2}-\int_{C} \frac{\mathrm{d} \lambda}{2 \pi \mathrm{i}} \frac{\mathrm{e}(-\lambda) \sigma(\lambda)}{1+\mathfrak{a}(\lambda \mid \kappa)} .
$$

The function $\sigma$ satisfies the linear integral equation

$$
\sigma(\lambda)=1+\int_{C} \frac{\mathrm{d} \mu}{2 \pi \mathrm{i}} \frac{K(\lambda-\mu) \sigma(\mu)}{1+\mathfrak{a}(\mu \mid \kappa)} .
$$

Its zero temperature limit

$$
\xi(\lambda)=\lim _{T \rightarrow 0+} \sigma(\lambda)
$$

is called the dressed charge. It plays an important role in the calculation of the asymptotics of correlation functions at $T=0$. For the lack of any better name we shall call $\sigma$, and also an $\alpha$-generalization of $\sigma$ to be considered below, the dressed charge as well.

Another possibility of expressing the magnetization per lattice site (6) is by means of a magnetization density $G$ satisfying

$$
G(\lambda)=\mathrm{e}(-\lambda)+\int_{C} \frac{\mathrm{d} \mu}{2 \pi \mathrm{i}} \frac{K(\lambda-\mu) G(\mu)}{1+\mathfrak{a}(\mu \mid \kappa)} .
$$

Applying the 'dressed function trick' to (7) and (9) we obtain

$$
m(h, T)=-\frac{1}{2}-\int_{C} \frac{\mathrm{d} \lambda}{2 \pi \mathrm{i}} \frac{G(\lambda)}{1+\mathfrak{a}(\lambda \mid \kappa)} .
$$

The integrability of the XXZ chain manifests itself in the existence of commuting families of transfer matrices and $Q$-operators of the associated six-vertex 
model. ${ }^{1}$ With an appropriate staggered choice of the horizontal spectral parameters the partition function of the six-vertex model on a rectangular lattice approximates the partition function of the XXZ chain. ${ }^{9}$ The approximation becomes exact in the so-called Trotter limit, when the extension of the lattice in vertical direction goes to infinity. By a modification of the boundary conditions in vertical direction we can also obtain an expression for the density matrix of a finite segment of the spin chain. ${ }^{5,6}$ The column-to-column transfer matrix in this approach is called the quantum transfer matrix. It satisfies a $t$ - $Q$-equation as well, which becomes a functional equation for the eigenvalues of the involved operators due to their commutativity.

We denote the dominant eigenvalue of the quantum transfer matrix by $\Lambda(\lambda \mid \kappa)$. This eigenvalue alone determines the free energy in the thermodynamic limit, when the horizontal extension of the lattice tends to infinity, $f(h, T)=$ $-T \ln \Lambda(0 \mid \kappa)$. Let the corresponding $Q$-function be $Q(\lambda \mid \kappa)$. Then $\Lambda$ and $Q$ satisfy the $t$ - $Q$-equation

$$
\Lambda(\lambda \mid \kappa) Q(\lambda \mid \kappa)=q^{\kappa} a(\lambda) Q(\lambda-\eta \mid \kappa)+q^{-\kappa} d(\lambda) Q(\lambda+\eta \mid \kappa),
$$

where $a(\lambda)$ and $d(\lambda)$ are the pseudo vacuum eigenvalues of the diagonal entries of the monodromy matrix associated with the quantum transfer matrix,

$$
a(\lambda)=\left(\frac{\operatorname{sh}\left(\lambda+\frac{\beta}{N}\right)}{\operatorname{sh}\left(\lambda+\frac{\beta}{N}-\eta\right)}\right)^{\frac{N}{2}}, \quad d(\lambda)=\left(\frac{\operatorname{sh}\left(\lambda-\frac{\beta}{N}\right)}{\operatorname{sh}\left(\lambda-\frac{\beta}{N}+\eta\right)}\right)^{\frac{N}{2}},
$$

and $\beta=2 J \operatorname{sh}(\eta) / T$.

Using the $Q$-functions corresponding to the dominant eigenvalue the auxiliary function $\mathfrak{a}$ can be expressed as

$$
\mathfrak{a}(\lambda \mid \kappa)=\frac{q^{-2 \kappa} d(\lambda) Q(\lambda+\eta \mid \kappa)}{a(\lambda) Q(\lambda-\eta \mid \kappa)} .
$$

In fact, the auxiliary function $\mathfrak{a}$ is usually defined by (13), and afterwards it is shown that $\mathfrak{a}$ satisfies the non-linear integral equation (1) in the Trotter limit. To be more precise, the $Q$-functions, the transfer matrix eigenvalue and the vacuum expectation values depend implicitly on the Trotter number $N$. Hence, $\mathfrak{a}$ as defined in (13) depends on $N$. One can show ${ }^{\mathrm{b}}$ that it satisfies the non-linear integral

\footnotetext{
${ }^{\mathrm{b}}$ For a recent pedagogical review on quantum spin chains within the quantum transfer matrix approach see, ${ }^{7}$ submitted to the same Festschrift volume for T. Miwa as this article.
} 
equation

$$
\begin{aligned}
& \ln \mathfrak{a}(\lambda \mid \kappa)=-2 \kappa \eta \\
& +\ln \left[\frac{\operatorname{sh}\left(\lambda-\frac{\beta}{N}\right) \operatorname{sh}\left(\lambda+\frac{\beta}{N}+\eta\right)}{\operatorname{sh}\left(\lambda+\frac{\beta}{N}\right) \operatorname{sh}\left(\lambda-\frac{\beta}{N}+\eta\right)}\right]^{\frac{N}{2}}-\int_{C} \frac{\mathrm{d} \mu}{2 \pi \mathrm{i}} K(\lambda-\mu) \ln (1+\mathfrak{a}(\mu \mid \kappa)) .
\end{aligned}
$$

Clearly this turns into (1) for $N \rightarrow \infty$. The integral equation (1) is the reason why the function $\mathfrak{a}$ is more useful for practical purposes than $Q$. It is hard to determine $Q$, and $Q$ has no simple Trotter limit. On the other hand, (1) determines a directly in the Trotter limit and can be converted into a form that can be accurately solved numerically.

Inserting (13) into (5) we obtain an expression for the dressed charge function in terms of logarithmic derivatives of $Q$-functions.

$$
\sigma(\lambda)=1+\frac{1}{2 \eta}\left(\frac{Q^{\prime}(\lambda-\eta \mid \kappa)}{Q(\lambda-\eta \mid \kappa)}-\frac{Q^{\prime}(\lambda+\eta \mid \kappa)}{Q(\lambda+\eta \mid \kappa)}\right),
$$

where the prime denotes the derivative with respect to $\kappa$. For the function $G$ defined in (9) no such simple expression in terms of $Q$-functions is known.

Below we shall introduce generalizations of the functions $\sigma$ and $G$ that depend on additional parameters. For the generalized dressed charge we will derive a generalization of (15). This will be used in a derivation of the asymptotic behaviour of the generalized magnetization density as a function of the spectral parameter.

\section{Linear integral equations}

It was shown in ${ }^{8}$ that all correlation functions of the $\mathrm{XXZ}$ chain regularized by a disorder parameter $\alpha$ can be expressed in terms of two functions, the ratio of eigenvalues

$$
\rho(\lambda)=\frac{\Lambda(\lambda \mid \kappa+\alpha)}{\Lambda(\lambda \mid \kappa)}
$$

and a function $\omega$ with the essential part $\Psi(\lambda, \mu)$ that can be characterized in terms of solutions of certain $\alpha$-dependent linear integral equations. ${ }^{2}$ A thorough understanding of these two functions is of fundamental importance for the further study of the correlation functions of the XXZ chain and for the application of the lattice results to quantum field theory in various scaling limits. ${ }^{3}$

We define the 'measure'

$$
\mathrm{d} m(\lambda)=\frac{\mathrm{d} \lambda}{2 \pi \mathrm{i} \rho(\lambda)(1+\mathfrak{a}(\lambda \mid \kappa))}
$$


and the deformed kernel

$$
K_{\alpha}(\lambda)=q^{-\alpha} \operatorname{cth}(\lambda-\eta)-q^{\alpha} \operatorname{cth}(\lambda+\eta) .
$$

Then, for $\nu$ inside $C$ the function $G$ is, by definition, the solution of the integral equation

$$
\begin{aligned}
& G(\lambda, \nu)= \\
& q^{-\alpha} \operatorname{cth}(\lambda-\nu-\eta)-\rho(\nu) \operatorname{cth}(\lambda-\nu)+\int_{C} \mathrm{~d} m(\mu) K_{\alpha}(\lambda-\mu) G(\mu, \nu) .
\end{aligned}
$$

Clearly $G$ is a generalization of the magnetization density (9) that depends on an additional spectral parameter and on the disorder parameter $\alpha$. For simplicity we keep the same notation also for the generalized function. $G$ enters the definition of $\Psi$ which, for $\nu_{1}, \nu_{2}$ inside $C$, is defined as

$$
\Psi\left(\nu_{1}, \nu_{2}\right)=\int_{C} \mathrm{~d} m(\mu) G\left(\mu, \nu_{2}\right)\left(q^{\alpha} \operatorname{cth}\left(\mu-\nu_{1}-\eta\right)-\rho\left(\nu_{1}\right) \operatorname{cth}\left(\mu-\nu_{1}\right)\right) .
$$

For $\nu$ or $\nu_{1}, \nu_{2}$ outside the contour, $G$ and $\Psi$ are given by the analytic continuations of the right hand side of (19) or (20), respectively.

Lemma 2.1. Asymptotic behaviour of $G$ and $\Psi$ as a functions of the spectral parameters.

(i)

$$
\lim _{\operatorname{Re} \lambda \rightarrow \infty} G(\lambda, \nu)=\lim _{\operatorname{Re} \nu \rightarrow \infty} G(\lambda, \nu)=0 .
$$

(ii)

$$
\begin{aligned}
\lim _{\operatorname{Re} \nu_{1} \rightarrow \infty} \Psi\left(\nu_{1}, \nu_{2}\right) & =-\frac{q^{-\alpha}-\rho\left(\nu_{2}\right)}{1+q^{2 \kappa}}, \\
\lim _{\operatorname{Re} \nu_{2} \rightarrow \infty} \Psi\left(\nu_{1}, \nu_{2}\right) & =-\frac{q^{\alpha}-\rho\left(\nu_{1}\right)}{1+q^{-2 \kappa}} .
\end{aligned}
$$

Proof. We may choose the contour $C$ as the rectangular contour of hight slightly less than $\gamma$ and of width $2 R$ depicted in figure $1 . R$ must be sufficiently large to include all Bethe roots. This is trivially possible for finite Trotter number, but also in Trotter limit $N \rightarrow \infty .^{7}$ Then the right hand side of (19) is holomorphic in $\lambda$ for $|\operatorname{Im} \lambda|<\gamma / 2$. It follows that

$$
\lim _{\operatorname{Re} \lambda \rightarrow \infty} G(\lambda, \nu)=q^{-\alpha}-\rho(\nu)-\left(q^{\alpha}-q^{-\alpha}\right) \int_{C} \mathrm{~d} m(\mu) G(\mu, \nu)=0 .
$$

Here the second equation will appear as lemma 2.3 below. We postpone the proof, because it needs some preparation. 
For the calculation of the asymptotics of $G$ for large $\operatorname{Re} \nu$ we have to take into account that $G(\lambda, \nu)$ as a function of $\lambda$ has pole at $\lambda=\nu$ with residue $-\rho(\nu)$. Hence, for $\nu$ outside $C$,

$$
\begin{aligned}
G(\lambda, \nu)=q^{-\alpha} \operatorname{cth}(\lambda-\nu-\eta)-\rho(\nu) \operatorname{cth}(\lambda-\nu) & \\
& -\frac{K_{\alpha}(\lambda-\nu)}{1+\mathfrak{a}(\nu \mid \kappa)}+\int_{C} \mathrm{~d} m(\mu) K_{\alpha}(\lambda-\mu) G(\mu, \nu) .
\end{aligned}
$$

Using that

$$
\lim _{\operatorname{Re} \nu \rightarrow \infty} \rho(\nu)=\frac{q^{\kappa+\alpha}+q^{-\kappa-\alpha}}{q^{\kappa}+q^{-\kappa}}, \quad \lim _{\operatorname{Re} \nu \rightarrow \infty} \mathfrak{a}(\nu \mid \kappa)=q^{-2 \kappa}
$$

and setting $g(\lambda)=\lim _{\nu \rightarrow \infty} G(\lambda, \nu)$ we obtain from (24)

$$
g(\lambda)=\int_{C} \mathrm{~d} m(\mu) K_{\alpha}(\lambda-\mu) g(\mu) .
$$

Then $g(\lambda)=0$, and (21) is proved.

A similar argument can be applied to prove (22b). For $\nu_{2}$ outside $C$ we have

$$
\begin{aligned}
\Psi\left(\nu_{1}, \nu_{2}\right) & =\int_{C} \mathrm{~d} m(\mu) G\left(\mu, \nu_{2}\right)\left(q^{\alpha} \operatorname{cth}\left(\mu-\nu_{1}-\eta\right)-\rho\left(\nu_{1}\right) \operatorname{cth}\left(\mu-\nu_{1}\right)\right) \\
& -\frac{1}{1+\mathfrak{a}\left(\nu_{2} \mid \kappa\right)}\left(q^{\alpha} \operatorname{cth}\left(\nu_{2}-\nu_{1}-\eta\right)-\rho\left(\nu_{1}\right) \operatorname{cth}\left(\nu_{2}-\nu_{1}\right)\right) .
\end{aligned}
$$

Using the second equation (21) and (25) we obtain (22b).

For the proof of (22a) we note that

$$
\begin{gathered}
\Psi\left(\nu_{1}, \nu_{2}\right)=\int_{C} \mathrm{~d} m(\mu) G\left(\mu, \nu_{2}\right)\left(q^{\alpha} \operatorname{cth}\left(\mu-\nu_{1}-\eta\right)-\rho\left(\nu_{1}\right) \operatorname{cth}\left(\mu-\nu_{1}\right)\right) \\
-\frac{G\left(\nu_{1}, \nu_{2}\right)}{1+\mathfrak{a}\left(\nu_{1} \mid \kappa\right)}
\end{gathered}
$$

if $\nu_{1}$ is outside $C$. Using (23) we conclude that

$$
\lim _{\nu_{1} \rightarrow \infty} \Psi\left(\nu_{1}, \nu_{2}\right)=-\frac{q^{\kappa}-q^{-\kappa}}{q^{\kappa}+q^{-\kappa}} \lim _{\nu_{1} \rightarrow \infty} G\left(\nu_{1}, \nu_{2}\right)-\frac{q^{-\alpha}-\rho\left(\nu_{2}\right)}{1+q^{2 \kappa}} .
$$

Thus, (22a) follows by means of (21).

Here a few comments are in order. For the correlation functions of the XXZ chain, ${ }^{2,8}$ it is actually not the function $\Psi$ but the closely related function $\omega^{\mathrm{c}}$ which

${ }^{\mathrm{c}}$ We follow here the notation of. ${ }^{3}$ In ref. ${ }^{2}$ a slightly different notation for $\omega$ was used. 
is at the heart of the theory

$$
\begin{aligned}
\omega\left(\nu_{1}, \nu_{2}\right)= & 2 \Psi\left(\nu_{1}, \nu_{2}\right) \mathrm{e}^{\alpha\left(\nu_{1}-\nu_{2}\right)}+ \\
& 4\left(\left(1+\rho\left(\nu_{1}\right) \rho\left(\nu_{2}\right)\right) g(\xi)-\rho\left(\nu_{1}\right) g\left(q^{-1} \xi\right)-\rho\left(\nu_{2}\right) g(q \xi)\right)
\end{aligned}
$$

where $g(\xi)=\Delta_{\xi}^{-1} \psi(\xi)$ with $\xi=\mathrm{e}^{\nu_{1}-\nu_{2}}$ should be understood as in (2.10) of ref. ${ }^{3}$ Using the explicit form of the function $\psi(\xi)=\frac{\xi^{\alpha}}{2} \frac{\xi^{2}+1}{\xi^{2}-1}$ and the above lemma (2.1) one can see that

$$
\lim _{\nu_{1} \rightarrow \pm \infty} \mathrm{e}^{-\alpha\left(\nu_{1}-\nu_{2}\right)} \omega\left(\nu_{1}, \nu_{2}\right)=0, \quad \lim _{\nu_{2} \rightarrow \pm \infty} \mathrm{e}^{-\alpha\left(\nu_{1}-\nu_{2}\right)} \omega\left(\nu_{1}, \nu_{2}\right)=0 .
$$

This is one of normalization conditions for $\omega$ introduced in. ${ }^{8}$

The asymptotics of $G$ and $\Psi$ with respect to the first argument was derived in. ${ }^{2}$ The reasoning there was also based on the second equation (23), which was obtained rather indirectly by means of the reduction property of the density matrix and a multiple integral representation for the six-vertex model with disorder parameter. Below we shall present a more direct proof of it in lemma 2.3, based on a representation of the generalized dressed charge in terms of $Q$-functions.

Perhaps the simplest proof of lemma 2.3 utilizes the symmetry ${ }^{2,8}$

$$
\Psi\left(\nu_{1}, \nu_{2} \mid \kappa, \alpha\right)=\Psi\left(\nu_{2}, \nu_{1} \mid-\kappa,-\alpha\right) .
$$

If we combine this with $\rho(\lambda \mid \kappa, \alpha)=\rho(\lambda \mid-\kappa,-\alpha)$, then (22a) follows from (22b). But (22a) inserted into (29) implies $\lim _{\nu_{1} \rightarrow \infty} G\left(\nu_{1}, \nu_{2}\right)=0$, and lemma 2.3 follows with the first equation (23).

Still, this is a little indirect and unsatisfactory. Here we are going for a more direct proof based upon the properties of the dressed charge function defined by

$$
\sigma(\lambda)=1+\int_{C} \mathrm{~d} m(\mu) \sigma(\mu) K_{\alpha}(\mu-\lambda) .
$$

Lemma 2.2. Dressed charge in terms of $Q$-functions.

$$
\sigma(\lambda)=\frac{q^{\alpha} \phi(\lambda-\eta)-q^{-\alpha} \phi(\lambda+\eta)}{\phi_{0}\left(q^{\alpha}-q^{-\alpha}\right)}
$$

where

$$
\phi(\lambda)=\frac{Q(\lambda \mid \kappa+\alpha)}{Q(\lambda \mid \kappa)}, \quad \phi_{0}=\operatorname{ch}\left(\int_{C} \frac{\mathrm{d} \lambda}{2 \pi \mathrm{i}} \ln \left(\frac{1+\mathfrak{a}(\lambda \mid \kappa+\alpha)}{1+\mathfrak{a}(\lambda \mid \kappa)}\right)\right) .
$$

Proof. We recall from the appendix of ${ }^{2}$ that

$$
\phi(\lambda)=\frac{Q(\lambda \mid \kappa+\alpha)}{Q(\lambda \mid \kappa)}=\prod_{j=1}^{N / 2} \frac{\operatorname{sh}\left(\lambda-\lambda_{j}(\kappa+\alpha)\right)}{\operatorname{sh}\left(\lambda-\lambda_{j}(\kappa)\right)},
$$


where the $\lambda_{j}$ are the Bethe roots of the dominant eigenstate of the quantum transfer matrix which are located inside the contour $C$. Due to the $t$ - $Q$-equation (11)

$$
\phi(\lambda)=\frac{q^{-\alpha} \phi(\lambda+\eta)}{\rho(\lambda)}+\frac{q^{\alpha} \phi(\lambda-\eta)-q^{-\alpha} \phi(\lambda+\eta)}{\rho(\lambda)(1+\mathfrak{a}(\lambda \mid \kappa))} .
$$

Here the first term on the right hand side is holomorphic inside $C$. Hence,

$$
\int_{C} \mathrm{~d} m(\mu)\left(q^{\alpha} \phi(\mu-\eta)-q^{-\alpha} \phi(\mu+\eta)\right) K_{\alpha}(\mu-\lambda)=\int_{C} \frac{\mathrm{d} \mu}{2 \pi \mathrm{i}} \phi(\mu) K_{\alpha}(\mu-\lambda) .
$$

The latter integral can be calculated. Note that

$$
\phi(\lambda+\mathrm{i} \pi)=\phi(\lambda), \quad K_{\alpha}(\lambda+\mathrm{i} \pi)=K_{\alpha}(\lambda)
$$

and

$$
\lim _{\operatorname{Re} \lambda \rightarrow \pm \infty} \phi(\lambda)=b^{ \pm 1}, \quad b=\exp \left(\sum_{j=1}^{N / 2}\left(\lambda_{j}(\kappa)-\lambda_{j}(\kappa+\alpha)\right)\right) .
$$

With the contours sketched in figure 1 it follows that

$$
\begin{aligned}
\int_{\widetilde{C}+C} \frac{\mathrm{d} \mu}{2 \pi \mathrm{i}} & \phi(\mu) K_{\alpha}(\mu-\lambda) \\
= & \int_{I_{2}+I_{4}} \frac{\mathrm{d} \mu}{2 \pi \mathrm{i}} \phi(\mu) K_{\alpha}(\mu-\lambda)=-\frac{b+b^{-1}}{2}\left(q^{\alpha}-q^{-\alpha}\right) \\
& =q^{-\alpha} \phi(\lambda+\eta)-q^{\alpha} \phi(\lambda-\eta)+\int_{C} \frac{\mathrm{d} \mu}{2 \pi \mathrm{i}} \phi(\mu) K_{\alpha}(\mu-\lambda) .
\end{aligned}
$$

Here we have used the periodicity (38) in the first equation, the fact that the integral is independent of $R$ and the asymptotics (39) in the second equation, and the fact that $\phi$ is free of poles inside $\widetilde{C}$ in the third equation. Setting $\phi_{0}=\left(b+b^{-1}\right) / 2$ and combining (37) and (40) we obtain (33).

It remains to show the second equation (34). It follows from

$\int_{C} \frac{\mathrm{d} \lambda}{2 \pi \mathrm{i}} \ln (1+\mathfrak{a}(\lambda \mid \kappa))=-\int_{C} \frac{\mathrm{d} \lambda}{2 \pi \mathrm{i}} \lambda \partial_{\lambda} \ln (1+\mathfrak{a}(\lambda \mid \kappa))=-\sum_{j=1}^{N / 2} \lambda_{j}(\kappa)-\beta / 2$,

and the proof is complete.

Clearly (33) turns into (15) in the limit $\alpha \rightarrow 0$. Equipped with lemma 2.2 we can now proceed with proving

Lemma 2.3. An identity for one-point functions.

$$
\int_{C} \mathrm{~d} m(\lambda) G(\lambda, \nu)=\frac{q^{-\alpha}-\rho(\nu)}{q^{\alpha}-q^{-\alpha}} .
$$




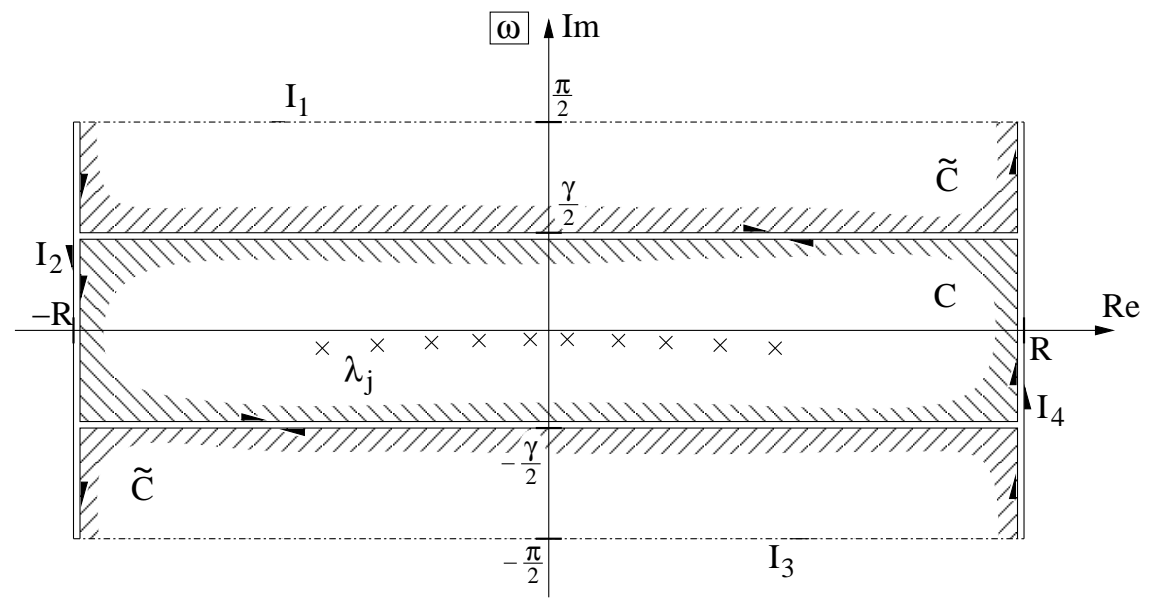

Fig. 1. Contours used in the proofs of lemma 2.2 and lemma 2.3.

Proof. First of all applying the dressed function trick to (19) and (32) we obtain

$$
\int_{C} \mathrm{~d} m(\lambda) G(\lambda, \nu)=\int_{C} \mathrm{~d} m(\lambda) \sigma(\lambda)\left(q^{-\alpha} \operatorname{cth}(\lambda-\nu-\eta)-\rho(\nu) \operatorname{cth}(\lambda-\nu)\right) \text {. }
$$

Then we insert (33) and (36) into the right hand side. It follows that

$$
\begin{aligned}
& \int_{C} \mathrm{~d} m(\lambda) G(\lambda, \nu)=\frac{1}{\phi_{0}\left(q^{\alpha}-q^{-\alpha}\right)} \\
& \quad \int_{C} \frac{\mathrm{d} \lambda}{2 \pi \mathrm{i}}\left(\phi(\lambda)-\frac{q^{-\alpha} \phi(\lambda+\eta)}{\rho(\lambda)}\right)\left(q^{-\alpha} \operatorname{cth}(\lambda-\nu-\eta)-\rho(\nu) \operatorname{cth}(\lambda-\nu)\right)= \\
& =\frac{1}{\phi_{0}\left(q^{\alpha}-q^{-\alpha}\right)}\left(q^{-\alpha} \phi(\nu+\eta)\right. \\
& \left.\quad+\int_{I_{2}+I_{4}-\widetilde{C}} \frac{\mathrm{d} \lambda}{2 \pi \mathrm{i}} \phi(\lambda)\left(q^{-\alpha} \operatorname{cth}(\lambda-\nu-\eta)-\rho(\nu) \operatorname{cth}(\lambda-\nu)\right)\right) \\
& =\frac{q^{-\alpha}-\rho(\nu)}{q^{\alpha}-q^{-\beta a}} .
\end{aligned}
$$

Here we have again referred to figure 1 in the second equation.

\section{Conclusions}

We would like to conclude with three more remarks. First, all the above remains valid if we consider a more general six-vertex model with a more general inhomogeneous choice of parameters in vertical direction. In that case the functions $a(\lambda)$ and $d(\lambda)$ in (12) and also the driving term in (14) have to be modified as in. ${ }^{2}$ 
Second, in reference ${ }^{2}$ the calculation of the $\operatorname{limit}_{\lim _{\nu_{1}} \rightarrow \infty} \Psi\left(\nu_{1}, \nu_{2}\right)$ was the only point, where we had to resort to a multiple integral representation, when we showed that the functions $\omega$ as defined in references ${ }^{2}$ and $^{8}$ are identical. With the proof presented in this note the approach to the correlation functions of the XXZ chain, based on the discovery of a hidden Grassmann symmetry, as developed in $^{2,4,8}$ becomes logically independent of the multiple integral representation that was also obtained in. ${ }^{2}$

Third, the function $\phi$ used above to express $\sigma$ seems quite interesting and may deserve further attention. Is it possible to express the solutions of other linear integral equations in terms of $\phi$ ? And is there a useful integral equation for $\phi$ itself? We hope we can come back to these questions in the future.

\section{Acknowledgment}

It a great pleasure and honour to dedicate this note to our dear friend Tetsuji Miwa on the occasion of his 60th birthday. We are grateful to Fedor Smirnov for suggesting us to prove lemma 2.3 directly. We wish to thank Alexander Seel for drawing the figure for us. Our work was generously supported by the Volkswagen foundation.

\section{References}

1. R. J. Baxter, Exactly Solved Models in Statistical Mechanics (Academic Press, London, 1982).

2. H. Boos and F. Göhmann, On the physical part of the factorized correlation functions of the XXZ chain, J. Phys. A 42 (2009) 315001.

3. H. Boos, M. Jimbo, T. Miwa and F. Smirnov, Hidden Grassmann structure in the XXZ model IV: CFT limit, preprint, arXiv:0911.3731 (2009).

4. H. Boos, M. Jimbo, T. Miwa, F. Smirnov and Y. Takeyama, Hidden Grassmann structure in the XXZ model II: creation operators, Comm. Math. Phys. 286 (2009) 875.

5. F. Göhmann, N. P. Hasenclever and A. Seel, The finite temperature density matrix and two-point correlations in the antiferromagnetic XXZ chain, J. Stat. Mech. (2005) P10015.

6. F. Göhmann, A. Klümper and A. Seel, Integral representations for correlation functions of the XXZ chain at finite temperature, J. Phys. A 37 (2004) 7625.

7. F. Göhmann and J. Suzuki, Quantum spin chains at finite temperature, preprint arXiv:1002.3194 (2010).

8. M. Jimbo, T. Miwa and F. Smirnov, Hidden Grassmann structure in the XXZ model III: introducing Matsubara direction, J. Phys. A 42 (2009) 304018.

9. A. Klümper, Thermodynamics of the anisotropic spin-1/2 Heisenberg chain and related quantum chains, Z. Phys. B 91 (1993) 507. 